\title{
Gastric neuroendocrine tumor treated with endoscopic submucosal dissection
}

\author{
Pawel Bojar ${ }^{1}$, Jaroslaw Swatek ${ }^{1}$, Jaroslaw Drabko ${ }^{2}$, Katarzyna Golec ${ }^{1}$, \\ Anna Ostrowska ${ }^{1}$, Justyna Szumilo ${ }^{1}$
}

\author{
${ }^{1}$ Department of Clinical Pathomorphology Medical University of Lublin, Jaczewskiego 8b, 20-090 Lublin, Poland \\ ${ }^{2}$ Gastromed Healthcare Center, Onyksowa 10, 20-582 Lublin, Poland
}

\section{ARTICLE INFO \\ Received 01 July 2016 \\ Accepted 26 August 2016}

\section{Keywords:}

neuroendocrine tumor, carcinoid,

endoscopic submucosal

dissection,

stomach.

\begin{abstract}
A case of a 59-year-old male patient with gastric neuroendocrine tumor which was misdiagnosed as adenocarcinoma, is presented. Herein, primary diagnosis was made due to the similarity of endoscopic pictures of both diseases and dues to the inappropriate interpretation of a small biopsy sample. The patient was qualified for endoscopic submucosal dissection. Microscopic examination of whole lesion, supplemented by immmunohistochemical reactions (chromogranin A, synaptophysin, cytokeratins 7 and 20, Ki67) revealed gastric neuroendocrine tumor (NET) G2.

The lesson learnt is that to provide effective treatment to the patient, it is necessary to use all available methods to make a proper diagnosis and to distinguish the suspected disease from others with similar features.
\end{abstract}

\section{INTRODUCTION}

Neuroendocrine tumors (NETs) arise from the system of endocrine cells that do not form separate endocrine glands, but are distributed singly or in small groups in various organs, mostly in the digestive system, bronchi and thyroid, or forming larger collections, as pancreatic islets, paraganglia and adrenal medulla. In the digestive system, based on morphological features and proliferative fraction, neuroendocrine neoplasms are divided into low-grade neuroendocrine tumors (NETs G1), intermediate-grade neuroendocrine tumors (NETs G2) and (high-grade) neuroendocrine carcinomas (NECs, G3). NETs G1 appear mainly in the small intestine and appendix, but can be found in the stomach and large intestine as well [1].

Although uncommon, gastric NETs have been diagnosed with increasing frequency because of the increasing number of upper gastrointestinal tract endoscopies performed [13]. Their endoscopic appearance is often uncharacteristic, and, sometimes, it is difficult to distinguish them from other lesions more common in this location. While endoscopic submucosal dissection is an approved treatment option in low-grade gastric NETs, there is little data concerning usage of this method in tumors other than of typical NET

\footnotetext{
* Corresponding author

e-mail: pawel.bojar@umlub.pl;

phone: +48 81 4486536; fax +48 814486531
}

G1 morphology. Thus, it is crucial to determine the nature of a tumor before treatment.

We present the case of a patient who underwent endoscopic submucosal dissection as a treatment for gastric NET G2 which was primarily misdiagnosed as adenocarcinoma.

\section{CASE DESCRIPTION}

A 59-year-old man with osteoarthritis was admitted to hospital because of macrocytic anemia $(\mathrm{Hgb}=9,3 \mathrm{~g} / \mathrm{dl}$, $\mathrm{MCV}=111 \mathrm{fl}$ ). Other biochemical tests revealed high C-reactive protein and high erythrocyte sedimentation rate, mildly elevated total bilirubin, as well as mildly elevated aspartate aminotransferase and alanine aminotransferase levels. Abdominal ultrasonography and computer tomography were performed, but revealed no relevant pathology. The patient underwent gastroscopy. A sessile polyp with central ulceration covered with fibrinous exudate was found in the gastric corpus (Fig. 1). Moreover, gastroscopic features of bile reflux were noted. The urease test was negative. Histological evaluation of biopsy samples obtained during the procedure revealed an ulcerated inflammatory polyp, focal intestinal metaplasia and infiltration of the submucosa by neoplastic cells positive in pancytokeratin immunostaining (CK AE1/AE3) and diagnosed as adenocarcinoma. On the basis of this diagnosis, the patient was qualified for endoscopic submucosal dissection. Pathological examination of 
the whole lesion showed a $0,65 \mathrm{~cm}$ submucosal tumor (Fig. 2A) composed of monotonous medium-sized cells with a "salt and pepper" chromatin appearance and small nucleoli. These presented the characteristic insular, trabecular and pseudoglandular patterns of growth (Fig. 2B-D). The tumor cells were also positive for neuroendocrine markers (chromogranin A and synaptophysin) (Fig. 2 A-B) and negative for cytokeratines 7 and 20 (Fig. 3 C-D). Furthermore, proliferative index (expression of Ki67) was 17\%, while the mitotic count was low (less than 1 mitosis per $10 \mathrm{HPF}$ ). Subsequently, a diagnosis of NET G2 was made. The lesion was totally removed with smallest resection margin about $0,05 \mathrm{~mm}$ (deep margin). The period after the procedure was uneventful. The patient is undergoing consecutive clinical evaluation for 18 months. Gastroscopic verification showed no signs of recurrence.

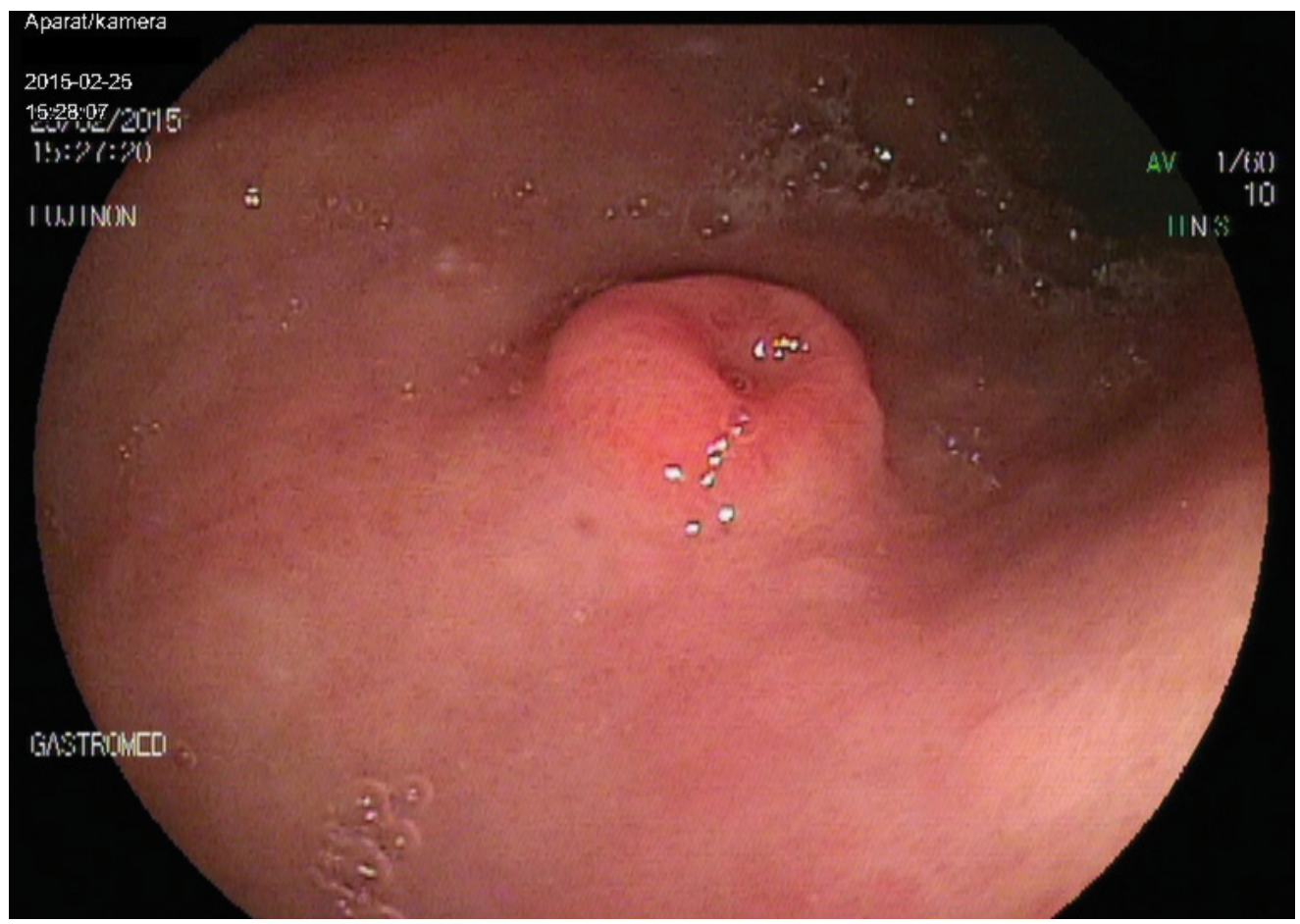

Figure 1. Gastric neuroendocrine tumor forming sessile polyp with central ulceration in the endoscopic examination
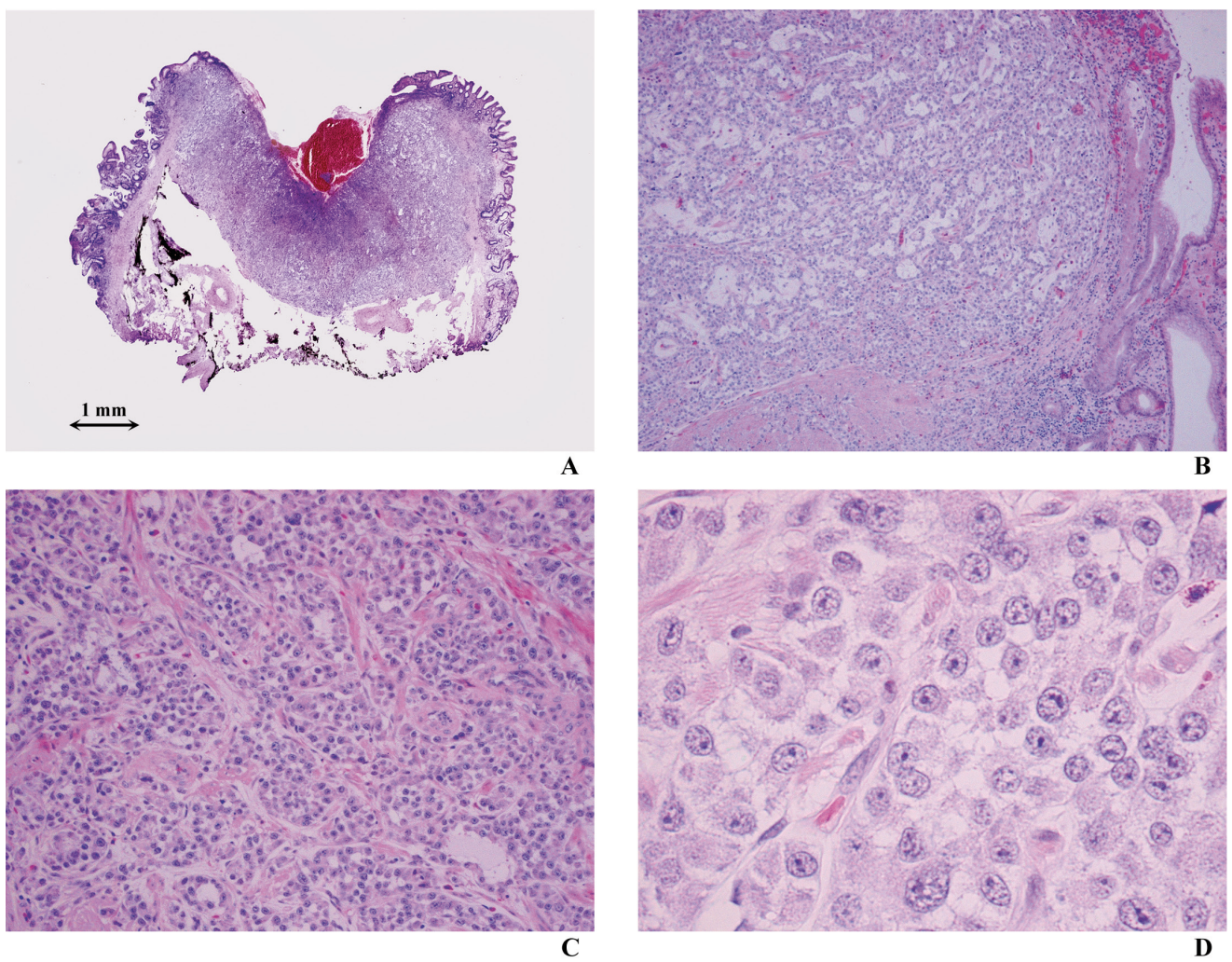

Figure 2. Completely removed neuroendocrine tumor (NET G2) located in gastric mucosa and submucosa (A) composed of monotonous medium-sized cells with insular and pseudoglandular growth patterns (B-D) (H\&E; A - macrophotography scale 1:1; objective magn. B - 10×, B - 20×, C - 40×) 

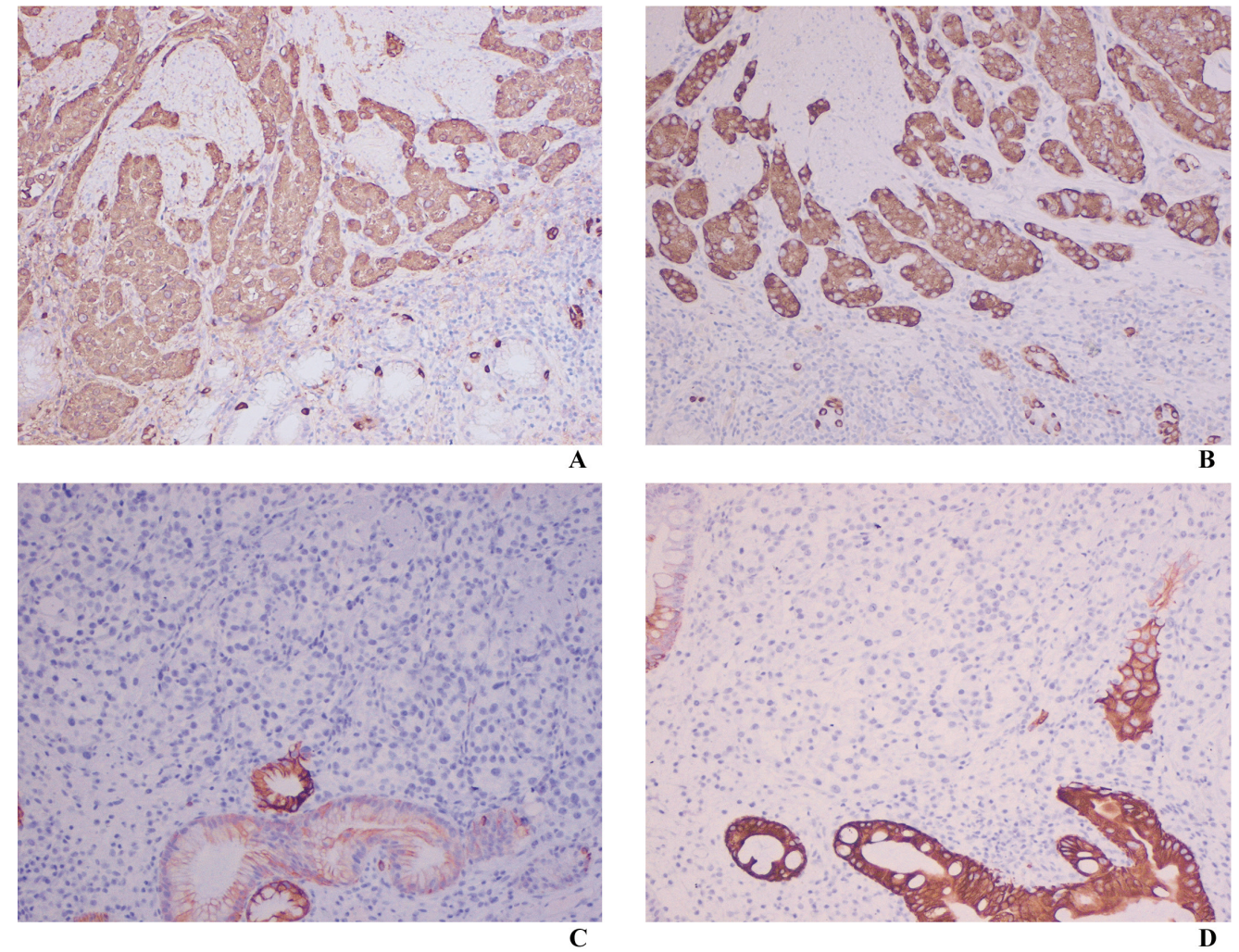

Figure 3. Positive immunostaining for chromogranin A (A) and synaptophisin (B) and negative for both cytokeratin 7 (C) and 20 (D) in gastric neuroendocrine tumor (Dako, EnVision FLEX; objective magn. A-D - 10×)

\section{DISCUSSION}

NETs are well known entities since 1907, when they were first described by Oberdorfer [8] and named carcinoid tumors. While they were considered as extremely rare in the pre-endoscopic era, nowadays, NETs represent up to $5 \%$ of all gastric tumors [5]. This result may also be associated with widespread and prolonged proton pump inhibitor treatment [5].

Diagnosis of NET based on endoscopic appearance and biopsy of the lesion is difficult. Most gastric NETs are small, round in shape, slightly elevated polyps, covered by normal mucosa, but some can be associated with central depression or ulceration in endoscopic evaluation - as seen in our case. Nevertheless, these features are not specific for NETs and there are variety of epithelial, mesenchymal or hamartomatous lesions of similar endoscopic picture. Endosonography is a helpful tool in making precise diagnosis before final surgical treatment [8].

While histopathological evaluation of the lesion is most important in establishing diagnosis, sampling by the forceps biopsy is often inadequate. That is why there is still unsatisfactory accuracy of biopsy evaluation, and why only polypectomy can provide definitive diagnosis [8]. Biopsy-based evaluation also can lead to diagnostic pitfalls, especially if NET was not included into the differential diagnosis. Positive immunohistochemical reaction with CK AE1/AE3is common, but of limited value when applied as a single immunostaining. While most NETs show expression of CK AE1/AE3, they usually lack expression of cytokeratin
7 and 20 [13]. Contrarily, most gastric adenocarcinomas, besides showing positivity for CK AE1/AE3, usually exhibit expression of CK7 (more often) or CK20 or both [9]. An important diagnostic feature of NET, is the expression of at least one neuroendocrine marker, such as chromogranin A, synaptophysin, or CD56 $[10,11]$. As these markers are not fully specific, the more endocrine markers that are expressed, the more likely it is that the tumor is of neuroendocrine origin. In our case positivity for two routinely used markers - chromogranin A and synaptophysin - was noted.

Features of diagnostic and prognostic significance in NETs are percentage of Ki67 positive cells and mitotic index. These allow the distinguishing of NET G1, NET G2 and neuroendocrine carcinoma (NEC). It should be noted that gastric NET are subdivided into three clinicopathological entities depending on pathogenesis and outcome [1]. Type I NET is associated with hypergastrinemia and chronic atrophic gastritis; type II - with multiple endocrine neoplasia type 1 (MEN1) and Zollinger-Ellison syndrome; type III is sporadic. It also must be underlined that there are differences in treatment of each type. While in type I and II, active surveillance and endoscopic or laparoscopic excision are accepted treatment options, type III NETs show a more aggressive natural course and usually need a surgical approach that is similar as in adenocarcinoma [12]. Nevertheless, some authors suggest that endoscopic mucosectomy or endoscopic submucosal dissection may be considered as initial treatment for patients with small type III NETs [6]. 


\section{CONCLUSIONS}

Successful treatment of neoplasms depends on many different factors, but a quick and proper diagnosis is probably of most importance. To be sure that the patient receives an effective therapy, it is necessary to use all available diagnostic tools, and to consider all possible diseases of similar morphology, symptoms and location. In a case of possible gastric NET, it is crucial to distinguish this from other epithelial, mesenchymal or hamartomatous lesions of similar endoscopic appearance - even if so-doing requires application of some additional diagnostic methods, such as endosonography and extended immunohistochemistry.

\section{REFERENCES}

1. Bosman F.T., Carneiro, R., Hruban H., Theise N.D., editors (2010), WHO Classification of Tumours of the Digestive System $4^{\text {th }}$ ed., Lyon: The International Agency for Research on Cancer.

2. Cai Y.C. et al.: Cytokeratin 7 and 20 and thyroid transcription factor 1 can help distinguish pulmonary from gastrointestinal carcinoid and pancreatic endocrine tumors. Hum.Pathol., 32, 10, 2001.

3. Erim T., Colak Y., Szomstein S.: Gastric carcinoid tumor after laparoscopic sleeve gastrectomy., Surgery for Obesity and Related Diseases, Volume 11, Issue 6, 2015.

4. Jianu C.S. et al.: Gastric carcinoids after long-term use of a proton pump inhibitor. Aliment.Pharmacol. Ther., 36, 7, 2012.
5. Kidd M., Gustafsson B., Modlin I.M.: Gastric carcinoids (neuroendocrine neoplasms). Gastroenterol.Clin. North. Am., 42, 2, 2013.

6. Kwon Y.H. et al.: Long-term follow up of endoscopic resection for type 3 gastric NET. World J.Gastroenterol., 19, 46, 2013.

7. Modlin I.M. et al.: Current status of gastrointestinal carcinoids. Gastroenterology. 128, 6, 2015.

8. Muehldorfer SM et al.: Multicenter Study Group "Gastric Polyps". Diagnostic accuracy of forceps biopsy versus polypectomy for gastric polyps: a prospective multicentre study. Gut, 50, 4, 2002.

9. Park S.Y. et al.: Expression of cytokeratins 7 and 20 in primary carcinomas of the stomach and colorectum and their value in the differential diagnosis of metastatic carcinomas to the ovary. Hum. Pathol., 33, 11, 2002.

10. Perryman S., Kaltenbach T., Eisenberg D.: Preoperative finding of gastric neuroendocrine tumor (gastric carcinoid) in a patient evaluated for bariatric surgery. Surgery for Obesity and RelatedDiseases, 7, 5, 2011.

11. Ramage J.K. et al.: UK and Ireland Neuroendocrine Tumour Society. Guidelines for the management of gastroenteropancreatic neuroendocrine (including carcinoid) tumours (NETs). Gut, 61, 1, 2012.

12. Wang Z. et al.: Retrospective analysis of the clinicopathological characteristics of gastrointestinal neuroendocrine neoplasms. Exp. Ther. Med., 10, 3, 2015.

13. Xu T.M. et al.: Clinicopathological features of primary gastric neuroendocrine neoplasms: A single-center analysis. J. Dig. Dis., $17,3,2016$ 